\title{
Neutrino Hierarchies from a Gauge Symmetry
}

\author{
Julian Heeck ${ }^{1,2}$, 田 and Werner Rodejohann ${ }^{1, \text { 团 }}$ \\ ${ }^{1}$ Max-Planck-Institut für Kernphysik, Saupfercheckweg 1, 69117 Heidelberg, Germany \\ ${ }^{2}$ Institute for Theoretical Physics, Kanazawa University, Kanazawa 920-1192, Japan
}

\begin{abstract}
We consider the phenomenology of the gauged abelian symmetry $B+3\left(L_{e}-L_{\mu}-L_{\tau}\right)$. Righthanded neutrinos necessary to cancel triangle anomalies are used in a type-I seesaw scheme to create active neutrino masses. Breaking the $B+3\left(L_{e}-L_{\mu}-L_{\tau}\right)$ symmetry spontaneously below the seesaw scale generates low energy neutrino mass matrices with the approximate symmetries $L_{e}$ (leading to normal hierarchy) or $L_{e}-L_{\mu}-L_{\tau}$ (inverted hierarchy). For the latter we need to introduce a $\mathbb{Z}_{2}$ symmetry which decouples one of the right-handed neutrinos. If exact, this $\mathbb{Z}_{2}$ leads to a Majorana dark matter candidate that interacts with the Standard Model via the $Z^{\prime}$ and a scalar $s$ originating from spontaneous breaking of the new symmetry. The measured relic abundance of the dark matter particle can be obtained around the scalar and $Z^{\prime}$ resonances, while direct detection experiments are mainly sensitive to scalar exchange, which is induced by mass mixing of $s$ with the standard Higgs.
\end{abstract}

PACS numbers: 14.60.Pq, 14.70.Pw, 12.60.-i, 14.60.St

Keywords: Neutrino Physics, Gauge Symmetry

\section{INTRODUCTION}

The observed neutrino mixing angles and mass-squared differences have launched an avalanche of models trying to explain their values. One possible starting point is the Majorana mass matrix in flavor basis, on which one then imposes symmetries. As far as continuous abelian symmetries of lepton numbers go, three interesting linear combinations have been identified for the zeroth-order approximation: $L_{e}$, $\bar{L} \equiv L_{e}-L_{\mu}-L_{\tau}$ and $L_{\mu}-L_{\tau}$, leading to normal hierarchy (NH), inverted hierarchy (IH) and quasidegeneracy in the neutrino mass spectrum [1]. The flavor structure of the mass matrices is

$$
\mathcal{M}_{\nu}^{L_{e}} \sim\left(\begin{array}{ccc}
0 & 0 & 0 \\
0 & \times & \times \\
0 & \times & \times
\end{array}\right), \quad \mathcal{M}_{\nu}^{\bar{L}} \sim\left(\begin{array}{ccc}
0 & \times & \times \\
\times & 0 & 0 \\
\times & 0 & 0
\end{array}\right), \quad \mathcal{M}_{\nu}^{L_{\mu}-L_{\tau}} \sim\left(\begin{array}{ccc}
\times & 0 & 0 \\
0 & 0 & \times \\
0 & \times & 0
\end{array}\right)
$$

where $\times$ denotes a non-zero entry. The last matrix conserving $L_{\mu}-L_{\tau}$ has the interesting property of being anomaly-free 2] (in the Standard Model (SM) with massless neutrinos), so the symmetry can be gauged, which leads to numerous interesting effects 3]. The other two symmetries have been considered as global symmetries [4] [) (or as an anomalous $U(1)$ [7]), but no effort has been put forward to construct a local version.

The reason why it is not easily possible to promote $L_{e}$ or $\bar{L}$ to a local symmetry with the SM particle content is due to arising quantum anomalies, even if we introduce SM-singlet right-handed neutrinos (RHNs). Extending the chiral fermion content of the model could serve as a viable way to cancel these triangle anomalies and construct a renormalizable Lagrangian. In the case of the above symmetries it can be shown that a complete fourth generation of fermions suffices to accomplish this task. ${ }^{1}$ However, the strict bounds on the fourth-generation fermions complicate model building severely, especially when it comes to the mass matrix of the - then four-active neutrinos.

A different way to cancel anomalies is the modification of the symmetry itself. For example, the quantum number $B-3 L_{e}$ is anomaly free in the SM plus RHNs [8], and leads to an $L_{e}$ symmetric neutrino mass matrix for the right-handed neutrinos (see Eq. (10). Models based on symmetries of the type $B-\sum_{\ell} x_{\ell} L_{\ell}$ and $\sum_{\ell} y_{\ell} L_{\ell}$ (with the constraints $\sum_{\ell} x_{\ell}=3$ and $\sum_{\ell} y_{\ell}=0$, respectively) have been discussed for example in Refs. [9 12]. Seesaw neutrino models with an additional $U(1)^{\prime}$ are also discussed in Ref. [13]. Some of the phenomenological aspects of such models (the scalar sector, dark

\footnotetext{
*Electronic address: julian.heeck@mpi-hd.mpg.de

${ }^{\dagger}$ Electronic address: werner.rodejohann@mpi-hd.mpg.de

1 This is easy to see because $L_{e}-L_{\mu}-L_{\tau}+L_{\ell_{4}}$ is a vectorlike symmetry in analogy to $L_{\alpha}-L_{\beta}$.
} 


\begin{tabular}{|l|l|l|}
\hline$L_{e}=\left(\begin{array}{l}\nu \\
e\end{array}\right)_{L} \sim(\mathbf{1}, \mathbf{2},-1)(3)$ & $e_{R}^{c} \sim(\mathbf{1}, \mathbf{1},+2)(-3)$ & $N_{i}^{c} \sim(\mathbf{1}, \mathbf{1}, 0)\left(Y^{\prime}\left(N_{i}^{c}\right)\right)$ \\
\hline$Q_{L}^{u}=\left(\begin{array}{l}u \\
d\end{array}\right)_{L} \sim\left(\mathbf{3}, \mathbf{2},+\frac{1}{3}\right)\left(+\frac{1}{3}\right)$ & $u_{R}^{c} \sim\left(\overline{\mathbf{3}}, \mathbf{1},-\frac{4}{3}\right)\left(-\frac{1}{3}\right)$ & $d_{R}^{c} \sim\left(\overline{\mathbf{3}}, \mathbf{1},+\frac{2}{3}\right)\left(-\frac{1}{3}\right)$ \\
\hline
\end{tabular}

Table I: $S U(3)_{C} \times S U(2)_{L} \times U(1)_{Y} \times U(1)_{B+3\left(L_{e}-L_{\mu}-L_{\tau}\right)}$ representations of left-handed SM fermions (only first generation shown) and right-handed neutrinos $N_{i}$. For the second and third generation the $U(1)_{B+3 \bar{L}}$ charge of the leptons changes sign.

matter candidates, etc.) are similar to frequently discussed $B-L$ analyses. However, choosing gauge groups that include flavor information makes it possible to provide predictions on neutrino mixing and mass spectrum, which is impossible in theories based solely on $B-L$. This interesting connection of flavor and gauge physics motivates us here to discuss the minimal gauged $B+3 \bar{L}$ model, which is free of anomalies if right-handed neutrinos with proper charges under the new $U(1)^{\prime}$ are introduced. Active neutrino masses are a result of a type-I seesaw mechanism, which is applicable only in the case of a broken symmetry, because the zeroth order right-handed neutrino mass matrix obeying $\bar{L}$ symmetry has rank 2. Interestingly, the resulting low energy neutrino mass matrix $\mathcal{M}_{\nu}$ does not necessarily obey a flavor structure resembling the one required from $\bar{L}$ conservation. Indeed, in what follows we will see that details of the breaking of $B+3\left(L_{e}-L_{\mu}-L_{\tau}\right)$ can lead to low energy neutrino physics with a normal or inverted hierarchy for the active neutrinos. As a possibility to force the inverted hierarchy in the active neutrino sector, we introduce a $\mathbb{Z}_{2}$ symmetry. Interestingly, the very same $\mathbb{Z}_{2}$ turns out to render one of the right-handed neutrino stable, and to become a dark matter candidate.

The paper is build up as follows: we will show the anomaly freedom of our symmetry in Sec. II] In Sec. III we show that the model can lead to either $L_{e}$ or $\bar{L}$ symmetric low-energy neutrino mass matrices via type-I seesaw, depending on the number of right-handed neutrinos and additional discrete symmetries. The $Z^{\prime}$ phenomenology of $B+3 \bar{L}$ has already been briefly considered in Ref. [14], as a special case of $B-\sum_{\ell} x_{\ell} L_{\ell}$, so we devote only a small section to its discussion (Sec. IV). Since the minimal scalar sector consists only of one additional complex scalar, the effects are well known from, e.g., minimal $B-L$ models. The resulting mixing among the scalars is briefly derived in Sec. $\mathrm{V}$. The discussion of the neutrino mass matrix naturally leads to an additional exchange symmetry, which-properly implemented — yields a stable right-handed neutrino as a dark matter candidate. We discuss the relic abundance of said dark matter candidate around the scalar and $Z^{\prime}$ resonances in Sec. VI] We conclude our findings in Sec. VII. The calculation of anomalies is presented in App. A while appendices B and C contain brief calculations that are not of utter importance to follow the main text.

\section{GAUGED $B+3\left(L_{e}-L_{\mu}-L_{\tau}\right)$ SYMMETRY}

We introduce $n_{N}$ right-handed neutrinos $N_{i}$ with $U(1)_{B+3 \bar{L}}$ quantum numbers $Y^{\prime}\left(N_{i}\right)$. The gauge group representations of the first-generation fermions are shown in Tab. I] for the second and third generation the $U(1)_{B+3 \bar{L}}$ charge of the leptons changes sign. Defining for simplicity $U(1)^{\prime} \equiv U(1)_{B+3 \bar{L}}$ and $Y^{\prime} \equiv B+3 \bar{L}$ we can calculate the triangle anomalies of the model. As shown in App. $\mathrm{A}$, the model is free of anomalies as long as the quantum numbers of the right-handed neutrinos satisfy

$$
\sum_{i}^{n_{N}} Y^{\prime}\left(N_{i}\right)=-3, \quad \sum_{i}^{n_{N}} Y^{\prime 3}\left(N_{i}\right)=-3^{3} .
$$

The minimal anomaly free model consists of only one right-handed neutrino with $\bar{L}$ charge -3 . There are no real solutions of Eq. (2) for $n_{N}=2$, but for odd $n_{N}$ we can choose $\bar{L}\left(N_{R, 1}\right)=-3$ and add pairs of righthanded neutrinos with arbitrary - but opposite - charge. Solutions without a charge \pm 3 , and therefore without Dirac coupling to the active neutrinos, can be obtained with five right-handed neutrinos, e.g. with the $\bar{L}$ charges $-1,2,-5,-5$ and 6 , respectively. It is clear that in this case $m_{D}=0=\mathcal{M}_{R}$, and hence the massless right-handed neutrinos decouple unless $\bar{L}$ is broken in a very specific way. Since this is cumbersome, we will restrict ourselves to RHNs with charges \pm 3 in the following.

The symmetry $U(1)_{B+3 \bar{L}}$ was already discussed in Ref. [14], where it is proposed as an origin for Rparity that also forbids proton decay via higher dimensional operators like $Q Q Q L$, which conserve $B-L$ but violate $B-\sum_{\ell} x_{\ell} L_{\ell}$ if $x_{\ell} \neq 1$. Since in Ref. [14] it is only briefly mentioned that the mass matrix for 
the right-handed neutrinos has no vanishing entries in the broken case, we feel it is still worth discussing the neutrino masses in more detail, due to their interesting structure.

It should be stressed that even though we are taking a non-supersymmetric model for simplicity, a similar discussion holds for the supersymmetric case of Ref. 14]. Supersymmetric particles aside, the main difference is the need for a second complex scalar (super-)field to fill the vanishing entries in the neutrino mass matrix. The model (superpotential, mass spectrum etc.) is then similar to supersymmetric $B-L$ models, which are intensively discussed in e.g. Refs. [15]. Assuming similar vacuum expectation values for both scalars makes the discussion of neutrino masses identical to Sec. III. The scalar and dark matter sectors will of course differ from Sec. $\mathrm{V}$ and Sec. VI in a supersymmetric context. For example, the mixing of the Higgs doublets $H_{i}$ and the new scalars $S_{i}$ will be severely suppressed [16], making the $Z^{\prime}$ boson the main mediator between the dark matter and SM sector. A discussion of dark matter (especially in the context of the additional $\mathbb{Z}_{2}$ symmetry that leads to inverted hierarchy) would be interesting, but lies outside the realm of this work.

\section{NEUTRINO MASSES}

In this section we discuss various interesting possibilities of the new gauge symmetry in the neutrino sector.

\section{A. Three right-handed neutrinos}

The most natural quantum number assignment for three right-handed neutrinos that cancels the anomalies of Eq. (2) is $+3,-3$ and -3 . After electroweak symmetry breaking, the Dirac and (symmetric) Majorana mass matrices for $\bar{\nu}_{i} N_{j}$ and $\bar{N}_{i}^{c} N_{j}$, respectively, take the form

$$
m_{D}=\left(\begin{array}{ccc}
a & 0 & 0 \\
0 & b & c \\
0 & d & e
\end{array}\right), \quad \mathcal{M}_{R}=\left(\begin{array}{ccc}
0 & X & Y \\
\cdot & 0 & 0 \\
\cdot & \cdot & 0
\end{array}\right) .
$$

As already mentioned in the introduction, the matrix $\mathcal{M}_{R}$ is singular, which means the usual seesaw formula $\mathcal{M}_{\nu} \simeq-m_{D} \mathcal{M}_{R}^{-1} m_{D}^{T}$ for the light neutrinos in the limit $X, Y \gg\left(m_{D}\right)_{i j}$ is not applicable. Instead of the $3 \nu_{\text {light }}+3 \nu_{\text {heavy }}$ scheme known from seesaw, the diagonalization of the full $6 \times 6$ matrix leads to the hierarchy $2 \nu_{\text {heavy }}+2 \nu_{\text {electroweak }}+2 \nu_{\text {light }}$, which is clearly not in agreement with experiments.

Since the model looks quite different after $U(1)^{\prime}$ breaking, let us introduce a complex scalar field $S \sim(\mathbf{1}, \mathbf{1}, 0)(+6)$ which acquires a vacuum expectation value (VEV). Collider limits on additional heavy neutral gauge bosons typically give limits $M_{Z^{\prime}} / g^{\prime} \sim\langle S\rangle \gtrsim 1-10 \mathrm{TeV}$ for the VEV, to be further discussed in Sec. IV $S$ couples to the right-handed neutrinos, so $\langle S\rangle$ fills all texture zeros in $\mathcal{M}_{R}$. As a result, $\mathcal{M}_{R}$ is in general an invertible matrix after $B+3 \bar{L}$ breaking:

$$
\mathcal{M}_{R}=\left(\begin{array}{ccc}
A & X & Y \\
\cdot & B & C \\
\cdot & \cdot & D
\end{array}\right), \quad \mathcal{M}_{R}^{-1}=-\frac{1}{\operatorname{det} \mathcal{M}_{R}}\left(\begin{array}{ccc}
C^{2}-B D & D X-C Y & B Y-C X \\
\cdot & Y^{2}-A D & A C-X Y \\
\cdot & \cdot & X^{2}-A B
\end{array}\right) .
$$

The scaling $X, Y \gg\langle S\rangle \gg\left(m_{D}\right)_{i j}$ leads to the order-of-magnitude structure of the low-energy neutrino mass matrix

$$
\mathcal{M}_{\nu} \simeq-m_{D} \mathcal{M}_{R}^{-1} m_{D}^{T} \sim\left(\begin{array}{ccc}
\varepsilon^{2} & \varepsilon & \varepsilon \\
\cdot & 1 & 1 \\
\cdot & \cdot & 1
\end{array}\right),
$$

with $\varepsilon \equiv\langle S\rangle / X$. Consequently, a low $B+3 \bar{L}$ breaking scale (compared to $\mathcal{M}_{R}$, not $m_{D}$ ) $\varepsilon \sim 0.1$ actually leads to a mass matrix that approximately conserves $L_{e}$ instead of $\bar{L}$ (see Eq. (1)). It is however not the most general $L_{e}$ symmetric matrix, because the zeroth-order mass matrix has the structure

$$
\mathcal{M}_{\nu} \sim\left(\begin{array}{ccc}
0 & 0 & 0 \\
\cdot & (c X-b Y)^{2} & (c X-b Y)(e X-d Y) \\
\cdot & \cdot & (e X-d Y)^{2}
\end{array}\right)+\mathcal{O}(\varepsilon),
$$


which gives only one massive neutrino $\nu_{3} \sim(c X-b Y) \nu_{\mu}+(e X-d Y) \nu_{\tau}$.

The solar mixing angle is still undefined at this order, due to an accidental $O(2)$ symmetry of the matrix (see Ref. [17]). Since the symmetry allows for mixing of $\mu$ and $\tau$, the charged lepton mass matrix is not diagonal in general and contributes to $\theta_{23}$. The atmospheric mixing angle will therefore be a combination of the charged-lepton mixing and the neutrino one

$$
\tan \theta_{23}^{\nu} \simeq \frac{c X-b Y}{e X-d Y}
$$

so we expect large but non-maximal mixing.

While not particularly predictive, we show the distribution of the mixing angles $\theta_{12}$ and $\theta_{13}$ in Fig. 1 (left). For these we generated random Yukawa couplings $\left|\left(m_{D}\right)_{i j}\right| \leq 1,|A|,|B|, \cdots<\varepsilon$ and $|X|,|Y|>1$ that lead to neutrino mixing parameters in their $3 \sigma$ range [18]. Here and in the following we restrict the parameters to real values for simplicity, resulting in vanishing CP-violating phases in the mixing matrix. In any case, since the Yukawa couplings can have arbitrary phases, we do not expect our model to be able to predict the $\mathrm{CP}$-violating phases. The solar angle tends to be large while the reactor angle $\theta_{13}$ is generally small, but in good agreement with the recent T2K [19], Double-Chooz [20], Daya Bay [21] and RENO 22] results of $\sin ^{2} \theta_{13} \simeq 0.025-0.03$.

The units of $m_{D}$ and $\mathcal{M}_{R}$ have not been specified yet, because they only fix the overall neutrino mass scale - and hence the $\Delta m_{i j}^{2}$ - but not the mixing angles. In the usual seesaw manner, the magnitude $m_{D}^{2} / \mathcal{M}_{R} \simeq 0.1 \mathrm{eV}$ does not fix the seesaw scale, but naturalness hints at a high scale.

Since the NH structure (5) has already been recognized before as a byproduct of the softly broken global $\bar{L}$ (see for example Refs. [5]), we will not attempt to redo all the calculations done before. Instead, we construct a model with a true $\bar{L}$ symmetry (and therefore inverted hierarchy) instead of the effective $L_{e}$ as above. It turns out we are just a $\mathbb{Z}_{2}$ symmetry away.

\section{B. Three right-handed neutrinos and $\mathbb{Z}_{2}$ symmetry}

The reason for the different approximate symmetries in $\mathcal{M}_{R}$ and $\mathcal{M}_{R}^{-1}$ is the occurring vanishing eigenvalue of $\mathcal{M}_{R}$ in the unbroken case. Since the number of right-handed neutrinos is fixed by anomaly cancellations to be odd, we cannot simply remove one of the $N_{i}$ to make $\mathcal{M}_{R}$ invertible. We can however forbid its coupling to all other particles by means of an additional discrete symmetry. We will discuss the simplest example below.

Defining an additional $\mathbb{Z}_{2}$ symmetry under which $N_{3}$ transforms as $N_{3} \rightarrow-N_{3}$ while all other fields are even, ${ }^{2}$ the only allowed interactions for $N_{3}$ are

$$
\begin{aligned}
\mathcal{L}_{N_{3}} & =i \overline{N_{3}} \gamma^{\mu}\left(\partial_{\mu}-i(-3) g^{\prime} Z_{\mu}^{\prime}\right) N_{3}-Y_{\chi} S{\overline{N_{3}}}^{c} N_{3}+\text { h.c. } \\
& =\frac{i}{2} \chi^{T} \mathcal{C} \gamma^{\mu} \partial_{\mu} \chi-\frac{3}{2} g^{\prime} Z_{\mu}^{\prime} \chi^{T} \mathcal{C} \gamma^{\mu} \gamma_{5} \chi-Y_{\chi} \frac{v_{S}}{\sqrt{2}} \chi^{T} \mathcal{C} \chi\left(1+\frac{s}{v_{S}}\right),
\end{aligned}
$$

making it stable and heavy after $B+3 \bar{L}$ breaking. In the last line we replaced the right-handed Dirac fermion $N_{3}$ by a Majorana fermion $\chi$ (see App. Cl) and used unitary gauge to make the $Z^{\prime}$ boson massive and eliminate $\mathfrak{I m}(S)$. The stable Majorana fermion $\chi$ is therefore a candidate for dark matter, to be further examined in Sec. VI] Note that the stability is due to the $\mathbb{Z}_{2}$, which was introduced to implement an inverted hierarchy for the active neutrinos.

The active neutrinos then couple only to $N_{1}$ and $N_{2}$, so at most two active neutrinos acquire mass at tree level. The $B+3 \bar{L}$ symmetry is broken in $\mathcal{M}_{R}$ by the parameters $A$ and $B$, so with the usual seesaw mechanism we find

$$
\mathcal{M}_{\nu} \simeq-\left(\begin{array}{ll}
a & 0 \\
0 & b \\
0 & c
\end{array}\right)\left(\begin{array}{ll}
A & X \\
X & B
\end{array}\right)^{-1}\left(\begin{array}{lll}
a & 0 & 0 \\
0 & b & c
\end{array}\right)=\frac{1}{X^{2}-A B}\left(\begin{array}{ccc}
a^{2} B & -a b X & -a c X \\
\cdot & b^{2} A & b c A \\
\cdot & \cdot & c^{2} A
\end{array}\right)
$$

\footnotetext{
2 This is equivalent to an exchange symmetry $N_{2} \leftrightarrow N_{3}$ as can be seen using the basis $\Psi_{1} \sim N_{2}+N_{3}, \Psi_{2} \sim N_{2}-N_{3}$.
} 

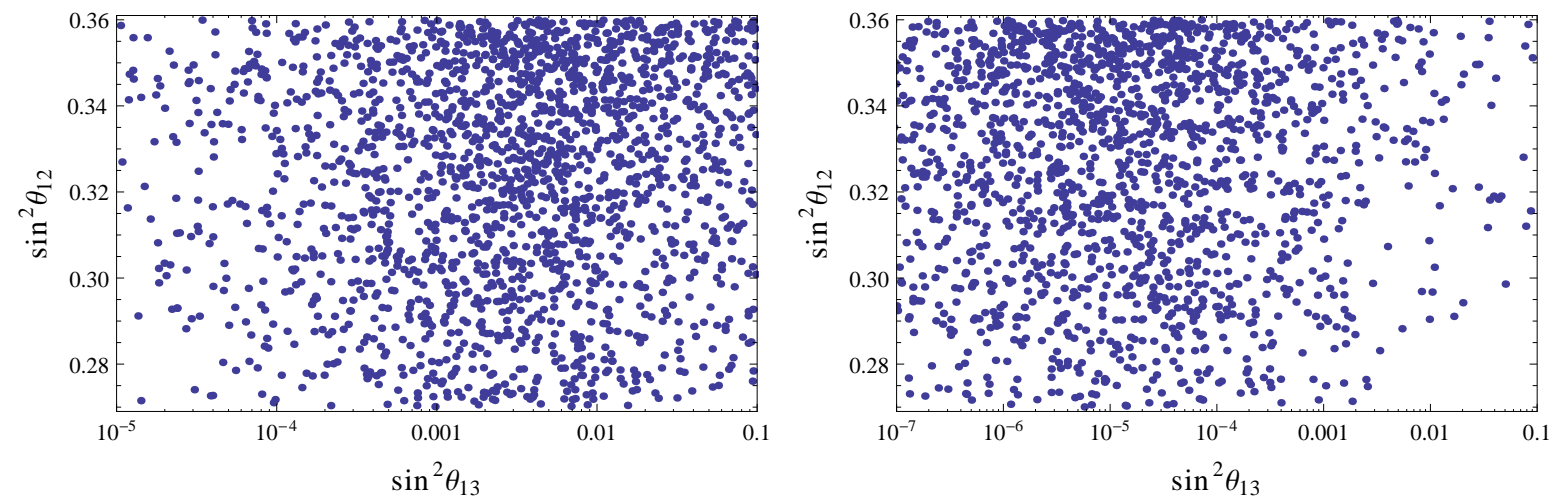

Figure 1: Left: scatter plots using the broken $B+3 \bar{L}$ low-energy neutrino mass matrix (5) $(\varepsilon=0.05)$ that leads to NH. Right: scatter plots using the neutrino mass matrix (10) $(\varepsilon=0.1)$ with five right-handed neutrinos and a $\mathbb{Z}_{2}$, which leads to IH. The accepted values of the mixing parameters satisfy the $3 \sigma$ bounds from Ref. [18], except for $\theta_{23}$, because it can be arbitrarily adjusted by the charged-lepton contribution.

which features an interesting structure [6, 23] : The decoupling of $N_{3}$ results in an invertible $\mathcal{M}_{R}^{2 \times 2}$, so $\mathcal{M}_{\nu}$ conserves $L_{e}-L_{\mu}-L_{\tau}$ in the limit $A, B \rightarrow 0$. This model also gives a simple explicit realization of "scaling" [24], seeing as the second and third column of $\mathcal{M}_{\nu}$ are proportional. Therefore we have an inverted hierarchy solution with $\theta_{13}=0$, whereas the atmospheric mixing angle is once again large but random, also due to the contributions of the charged leptons. At 2-loop level radiative corrections induce a non-zero $\theta_{13}$, but of practically irrelevant magnitude 25]. The solar mixing angle becomes maximal for $A, B \rightarrow 0$, so the breaking scale needs to be close to the bare mass terms to lower $\theta_{12}$.

Since a vanishing reactor angle is by now excluded at $\sim 6 \sigma$, we have to modify our model to make it phenomenologically viable. Here, $\theta_{13}$ and the mass of the lightest neutrino are linked [24], so we need to break $\mathbb{Z}_{2}$ to couple $N_{3}$ to the active neutrinos if we want $\theta_{13} \neq 0$. Therefore, a non-zero $\theta_{13}$ will lead to an unstable DM candidate $\chi$, with a short lifetime compared to the age of the Universe in general (see App. B for an estimate). Note that an explicit (soft) $\mathbb{Z}_{2}$ breaking by the coupling $\bar{N}_{1}^{c} N_{3}$ does not lead to IH, but rather an $L_{e}$ symmetric $\mathcal{M}_{\nu}$ (11). Correspondingly, the scalar sector needs to be enlarged quite a bit to achieve IH with non-vanishing $\theta_{13}$, which is why we will not discuss this model any further. Without touching the $\mathbb{Z}_{2}$ symmetry we could of course introduce a Higgs triplet (type-II seesaw) to generate $\theta_{13} \neq 0$, but once again the scalar sector blows up. Another solution would be the introduction of additional scalar doublets - charged under $U(1)^{\prime}$ - which generate off-diagonal mass terms for the charged leptons and consequently modify the PMNS mixing matrix. Obviously this once again complicates the scalar sector of the model and will therefore not be discussed further.

In the next section we will show that an extension of the fermion sector can easily generate a nonvanishing reactor angle while maintaining a simple scalar sector and the exact $\mathbb{Z}_{2}$ symmetry.

\section{Five right-handed neutrinos and $\mathbb{Z}_{2}$ symmetry}

Since the extension by scalars is cumbersome, we seek out a different solution to generate $\theta_{13} \neq 0$. The anomaly condition (2) can be fulfilled for five right-handed neutrinos with $B+3 \bar{L}$ charges $+3,+3$, $-3,-3$, and -3 , respectively. To obtain an invertible $\mathcal{M}_{R}$-and therefore an approximate $\bar{L}$ symmetric $\mathcal{M}_{\nu}$ - we once again decouple one of the right-handed neutrinos $\left(\chi \equiv N_{3}\right)$ by imposing a $\mathbb{Z}_{2}$ symmetry. Again, $\chi$ will be our dark matter candidate, to be discussed in Sec. VI.

After symmetry breaking with the scalars $H \sim(\mathbf{1}, \mathbf{2},+1)(0)$ and $S \sim(\mathbf{1}, \mathbf{1}, 0)(+6)$ we obtain the mass matrix

$$
\mathcal{M}_{\nu} \simeq-\left(\begin{array}{llll}
a & b & 0 & 0 \\
0 & 0 & c & d \\
0 & 0 & e & f
\end{array}\right)\left(\begin{array}{cc}
\mathcal{A} & \mathcal{X} \\
\mathcal{X}^{T} & \mathcal{B}
\end{array}\right)^{-1}\left(\begin{array}{lll}
a & 0 & 0 \\
b & 0 & 0 \\
0 & c & e \\
0 & d & f
\end{array}\right)
$$


where $\mathcal{X}$ is an arbitrary $2 \times 2$ matrix (the gauge invariant mass terms for the right-handed neutrinos) and $\mathcal{A}, \mathcal{B}$ are symmetric $2 \times 2$ matrices generated by spontaneous $B+3 \bar{L}$ breaking. For $c f-e d \neq 0$ there is no massless neutrino $\alpha \nu_{\mu}+\beta \nu_{\tau}$, so we have $\theta_{13} \neq 0$ in general. The solar mixing angle becomes maximal for $\mathcal{A}, \mathcal{B} \rightarrow 0$, so the breaking scale needs to be close to the bare mass terms to lower $\theta_{12}$. A large $\theta_{13}$ in agreement with recent results also forbids a too low breaking scale, meaning that the breaking parameter should be at least $\varepsilon \simeq 0.1$ in our minimal model. For the scatter plots in Fig. 1 (right) we generated random Yukawa couplings $\left|\left(m_{D}\right)_{i j}\right| \leq 1,\left|(\mathcal{A})_{i j}\right|,\left|(\mathcal{B})_{i j}\right| \leq \varepsilon$ and $\left|(\mathcal{X})_{i j}\right|>1$. Except for the approximate $\bar{L}$ symmetry in the limit $\mathcal{A}_{i j}, \mathcal{B}_{i j} \ll \mathcal{X}_{m n}$ (and the corresponding inverted hierarchy) there is no further structure in $\mathcal{M}_{\nu}$, so we refrain from any analytical discussion.

We conclude the section by stressing once more that the spontaneously broken $B+3 \bar{L}$ symmetry can provide mass matrices for either normal or inverted hierarchy, just depending on whether the number of "active" right-handed neutrinos is odd or even, respectively. Since anomaly cancellation requires an odd number of $N_{i}$ - at least for physically interesting charge assignments - the decoupling to get IH needs to be imposed by additional symmetries, which can easily lead to stable dark matter candidates. While we discussed only the simplest decoupling symmetry $\mathbb{Z}_{2}$, one can of course implement a more elaborate structure on this sub-sector.

\section{GAUGE SECTOR}

In this section we will briefly discuss constraints on the neutral gauge boson of the gauged $B+3 \bar{L}$ symmetry and possible detection prospects. The presented results are independent of the neutrino sector. Extending the gauge group of the SM $G_{\mathrm{SM}} \equiv S U(3)_{C} \times S U(2)_{L} \times U(1)_{Y}$ by $U(1)^{\prime}$ leads to possible $Z-Z^{\prime}$ mixing, either from the VEV of a scalar in a non-trivial representation of $S U(2)_{L} \times U(1)_{Y}$ and $U(1)^{\prime}$ or via the kinetic mixing angle $\chi$ that connects the $U(1)$ field strength tensors 26]. The relevant Lagrange density $\mathcal{L}=\mathcal{L}_{\mathrm{SM}}+\mathcal{L}_{Z^{\prime}}+\mathcal{L}_{\text {mix }}$ after breaking $S U(2)_{L} \times U(1)_{Y} \times U(1)^{\prime}$ to $U(1)_{\mathrm{EM}}$ then consists of:

$$
\begin{aligned}
\mathcal{L}_{\mathrm{SM}} & =-\frac{1}{4} \hat{B}_{\mu \nu} \hat{B}^{\mu \nu}-\frac{1}{4} \hat{W}_{\mu \nu}^{a} \hat{W}^{a \mu \nu}+\frac{1}{2} \hat{M}_{Z}^{2} \hat{Z}_{\mu} \hat{Z}^{\mu}-\frac{\hat{e}}{\hat{c}_{W}} j_{Y}^{\mu} \hat{B}_{\mu}-\frac{\hat{e}}{\hat{s}_{W}} j_{S U(2)}^{a \mu} \hat{W}_{\mu}^{a}, \\
\mathcal{L}_{Z^{\prime}} & =-\frac{1}{4} \hat{Z}_{\mu \nu}^{\prime} \hat{Z}^{\prime \mu \nu}+\frac{1}{2} \hat{M}_{Z^{\prime}}^{2} \hat{Z}_{\mu}^{\prime} \hat{Z}^{\prime \mu}-\hat{g}^{\prime} j^{\prime \mu} \hat{Z}_{\mu}^{\prime}, \\
\mathcal{L}_{\text {mix }} & =-\frac{\sin \chi}{2} \hat{Z}^{\prime \mu \nu} \hat{B}_{\mu \nu}+\delta \hat{M}^{2} \hat{Z}_{\mu}^{\prime} \hat{Z}^{\mu} .
\end{aligned}
$$

Since the above gauge eigenstates have a non-diagonal mass matrix and kinetic terms, the physical mass eigenstates are linear combinations of the hatted fields. Setting for simplicity the kinetic mixing angle $\chi$ to zero, the transformation to the mass eigenstates $Z_{1}$ and $Z_{2}$ takes the simple form

$$
\left(\begin{array}{c}
Z_{1} \\
Z_{2}
\end{array}\right)=\left(\begin{array}{cc}
\cos \theta & \sin \theta \\
-\sin \theta & \cos \theta
\end{array}\right)\left(\begin{array}{c}
\hat{Z} \\
\hat{Z}^{\prime}
\end{array}\right), \quad \tan 2 \theta=\frac{2 \delta \hat{M}^{2}}{\hat{M}_{Z}^{2}-\hat{M}_{Z^{\prime}}^{2}}
$$

which modifies the couplings of the gauge bosons to fermions (see Ref. [27] for more details). Using a modified version of GAPP [28] to fit our model with an arbitrary scalar sector we obtain the $95 \%$ C.L. limit $\left|g^{\prime} \sin \theta\right| \lesssim 10^{-4}$ (see Fig. 2) from electroweak precision data. Constraints for the mass $M_{Z^{\prime}}$ are obtained from collider searches, as the gauge boson of $U(1)_{B+3 \bar{L}}$ couples directly to first-generation particles. LEP-2 searches for new physics give a stronger limit than Tevatron, namely $M_{Z^{\prime}} / g^{\prime} \gtrsim 13.5 \mathrm{TeV}$ at $95 \%$ C.L. 29], because the $Z^{\prime}$ couples strongly to the electron $\left(Y^{\prime}(e)=3\right)$.

In the following we will ignore any $Z-Z^{\prime}$ mixing, be it mass mixing (not induced at tree-level in our minimal model) or kinetic mixing; with $\mathcal{L}_{\text {mix }}=0$ we can omit all the hats of the parameters in Eq. (11).

With $n_{N}$ heavy neutrinos below $M_{Z^{\prime}}$-and with charges $\left|Y^{\prime}(N)\right|=3$-we can calculate the $Z^{\prime}$ width:

$$
\begin{aligned}
\Gamma\left(Z^{\prime} \rightarrow f \bar{f}\right) & \simeq \frac{g^{\prime 2}}{12 \pi} M_{Z^{\prime}}\left(3 Y_{\nu}^{\prime 2} / 2+n_{N} Y_{N}^{\prime 2} / 2+3 Y_{\ell}^{\prime 2}+3 N_{c} Y_{u}^{\prime 2}+3 N_{c} Y_{d}^{\prime 2}\right) \\
& =\frac{g^{\prime 2}}{24 \pi} M_{Z^{\prime}}\left(85+27 n_{N}\right)
\end{aligned}
$$

with the number of colors $N_{c}=3$. The main contribution comes from the leptons, because of the large $B+3 \bar{L}$ charge, which can be used to distinguish this model from the similar $B-L$ model at colliders. The 


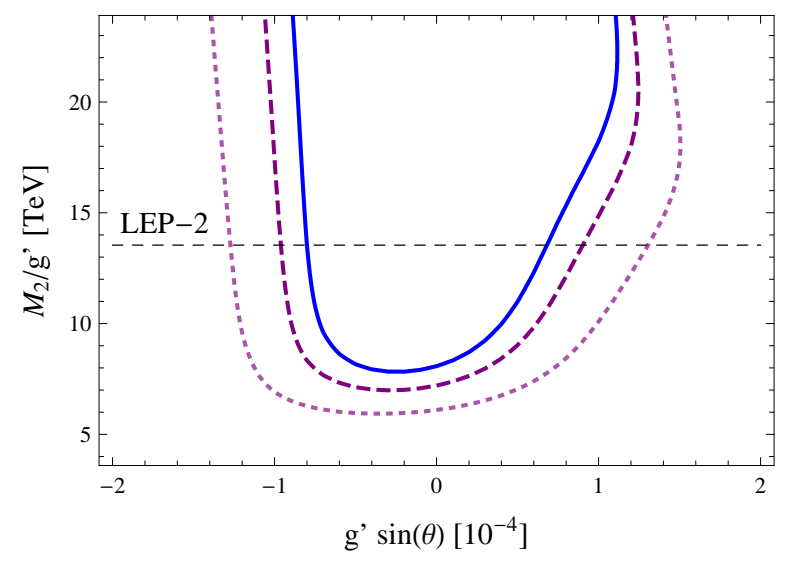

Figure 2: $\chi^{2}$ contours $\left(90 \%, 95 \%\right.$ and $99 \%$ C.L.) in the $M_{2}$-sin $(\theta)$ plane. The horizontal dashed line is the $95 \%$ C.L. lower limit from LEP-2 [29].

prospects of detecting the heavy $Z^{\prime}$ at the LHC were discussed in Ref. [14]; for $g^{\prime}=0.1$ the final stage of the $\mathrm{LHC}\left(\sqrt{s}=14 \mathrm{TeV}\right.$, integrated luminosity $\left.L \simeq 100 \mathrm{fb}^{-1}\right)$ can probe the model up to $M_{Z^{\prime}} \simeq 3.6 \mathrm{TeV}$ via the dilepton $Z^{\prime}$ resonance.

We note that the non-universal lepton coupling of $B+3 \bar{L}$ gives rise to non-standard neutrino interactions (NSIs), which are usually parameterized by the non-renormalizable effective Lagrangian

$$
\mathcal{L}_{\mathrm{NSI}}^{\mathrm{eff}}=-2 \sqrt{2} G_{F} \varepsilon_{\alpha \beta}^{f P}\left[\bar{f} \gamma^{\mu} P f\right]\left[\bar{\nu}_{\alpha} \gamma_{\mu} P_{L} \nu_{\beta}\right]
$$

in our case obtained upon integrating out the heavy gauge boson $Z^{\prime}$. Without going into details, we can estimate

$$
\varepsilon_{\alpha \beta} \sim \frac{v_{E W}^{2}}{\left(M_{Z^{\prime}} / g^{\prime}\right)^{2}} \operatorname{diag}(1,-1,-1)=\frac{v_{E W}^{2}}{\left(M_{Z^{\prime}} / g^{\prime}\right)^{2}} \operatorname{diag}(2,0,0)+\frac{v_{E W}^{2}}{\left(M_{Z^{\prime}} / g^{\prime}\right)^{2}} \operatorname{diag}(1,1,1) .
$$

The magnitude is very small $\left(\varepsilon \sim 10^{-4}\right)$ and since the term proportional to the identity matrix does not affect oscillations, we actually only induce $\varepsilon_{e e}$, i.e. modify the usual matter potential, which is hard to measure.

\section{MINIMAL SCALAR SECTOR}

In this section we will discuss the scalar sector of the theory, which is again independent on the neutrino physics. In addition to the usual scalar doublet $H \sim(\mathbf{1}, \mathbf{2},+1)(0)$ of the SM, we introduce a complex scalar $S \sim(\mathbf{1}, \mathbf{1}, 0)(+6)$ that will break the $B+3 \bar{L}$ symmetry spontaneously. The discussion is analogous to the highly discussed minimal $B-L$ scalar sector [30]. The potential has the simple form

$$
V(H, S)=-\mu_{1}^{2}|H|^{2}+\lambda_{1}|H|^{4}-\mu_{2}^{2}|S|^{2}+\lambda_{2}|S|^{4}+\delta|S|^{2}|H|^{2},
$$

where we assume $\mu_{i}^{2}>0$ to generate VEVs $v \equiv \sqrt{2}|\langle H\rangle|$ and $v_{S} \equiv \sqrt{2}|\langle S\rangle|$. The positivity of the potential gives the constraints $\lambda_{i}>0$ and $\lambda_{1} \lambda_{2}>\delta^{2} / 4$. In unitary gauge the charged component of $H$ is absorbed by $W^{ \pm}$, the pseudoscalar neutral component by $Z$, and the pseudoscalar component of $S$ by $Z^{\prime}$, hence we may go to the physical basis $H \rightarrow(0,(h+v) / \sqrt{2})^{T}, S \rightarrow\left(s+v_{S}\right) / \sqrt{2}$, which after the replacement of $\mu_{i}^{2}$ by the VEVs gives the potential:

$$
\begin{aligned}
V(h, s)= & \lambda_{1} v^{2} h^{2}+\lambda_{2} v_{S}^{2} s^{2}+\delta v v_{S} h s \\
& +\lambda_{1} v h^{3}+\frac{\lambda_{1}}{4} h^{4}+\lambda_{2} v_{S} s^{3}+\frac{\lambda_{2}}{4} s^{4}+\frac{\delta}{4} h^{2} s^{2}+\frac{\delta}{2} v h s^{2}+\frac{\delta}{2} v_{S} h^{2} s .
\end{aligned}
$$

The resulting mass matrix for the neutral scalars $h$ and $s$

$$
\mathcal{M}_{\text {scalar }}^{2}=\left(\begin{array}{cc}
2 \lambda_{1} v^{2} & \delta v v_{S} \\
\delta v v_{S} & 2 \lambda_{2} v_{S}^{2}
\end{array}\right)
$$


leads to the mass eigenstates $\phi_{1}$ and $\phi_{2}$ :

$$
\left(\begin{array}{c}
\phi_{1} \\
\phi_{2}
\end{array}\right)=\left(\begin{array}{cc}
\cos \alpha & -\sin \alpha \\
\sin \alpha & \cos \alpha
\end{array}\right)\left(\begin{array}{l}
h \\
s
\end{array}\right), \quad \tan 2 \alpha=\frac{\delta v v_{S}}{\lambda_{2} v_{S}^{2}-\lambda_{1} v^{2}}
$$

with the masses $m_{1,2}^{2}=\lambda_{1} v^{2}+\lambda_{2} v_{S}^{2} \mp \sqrt{\left(\lambda_{2} v_{S}^{2}-\lambda_{1} v^{2}\right)^{2}+\delta^{2} v_{S}^{2} v^{2}}$. In the limit $v_{S} \gg v$ we obtain $\alpha \simeq \delta v / 2 \lambda_{2} v_{S}$ and $m_{1}^{2} \simeq 2\left(\lambda_{1}-\delta^{2} / 4 \lambda_{2}\right) v^{2}$, so the Higgs mass is reduced compared to the SM.

The LEP-2 bounds on $M_{Z^{\prime}} / g^{\prime}$ translate into the constraint $v_{S}>2.3 \mathrm{TeV}$, which is to be compared to the VEV in minimal $B-L$ models $v_{B-L}>3-3.5 \mathrm{TeV}$. The masses of $Z^{\prime}, \phi_{2}$ and $\chi$ can of course be smaller, since they involve additional coupling constants (that are completely independent of each other):

$$
M_{Z^{\prime}}=6 g^{\prime} v_{S}, \quad m_{2} \simeq m_{s} \simeq \sqrt{2 \lambda_{2}} v_{S}, \quad M_{\chi}=\sqrt{2} Y_{\chi} v_{S} .
$$

Seeing as the VEV $v_{S}$ is connected to the seesaw scale (Eqs. (5), (9) ) one could also consider $v_{S} \sim$ $10^{15} \mathrm{GeV}$, which would make $Z^{\prime}$ and $s$ pretty much impossible to observe. We will therefore focus on the low-energy end of the seesaw scale, which can lead to observable effects. The arising effects are nearly identical to the highly discussed minimal $B-L$ scalar sector [30], the main difference being a larger $Z^{\prime}$ coupling to leptons, right-handed neutrinos and the scalar $s$; for these particles, $B-L$ results can be translated via $g^{\prime} \rightarrow 3 g^{\prime}$, while the coupling strength to quarks does not change. A future lepton collider would therefore be the ideal machine to test this model and distinguish it from $B-L$ by the decay products of the $Z^{\prime}$ resonance.

\section{DARK MATTER}

As we have seen in the previous sections, our model leads to a stable right-handed neutrino $\chi$ which interacts with $Z^{\prime}$ and the $\phi_{i}$ via the Lagrangian from Eq. (8). The measured relic density [31] $\Omega_{\chi} h^{2}=$ $0.1123 \pm 0.0035$ can be obtained around either of the scalar $s$-channel resonances $M_{\chi} \simeq m_{i} / 2$, but for the $\phi_{1}$-resonance one needs a large mixing angle $\alpha$. Choosing parameters that make the model testable at LHC and direct DM detection experiments $-M_{\chi} \sim 10-100 \mathrm{GeV}, m_{2} \sim 100 \mathrm{GeV}$ - can lead to viable DM relic abundance in complete analogy to Ref. [32], where a $\mathbb{Z}_{2}$ symmetry is added to the minimal $B-L$ model to make one of the right-handed neutrinos stable. We stress however that the $\mathbb{Z}_{2}$ in our model was not introduced to make a particle stable, but to generate the right flavor symmetry in the neutrino mass matrix. The stability of $\chi$ is in that sense just a welcome accident. ${ }^{3}$ We show the relic abundance of $\chi$ as a function of its mass and the $h-s$ mixing angle $\alpha$ in Fig. 3, as calculated with a modified version of microMEGAs [33]. There is no difference between the $B+3 \bar{L}$ model and the $B-L$ model in the region $M_{\chi} \ll M_{Z^{\prime}}$ of parameter space, because the $Z^{\prime}$ plays a sub-dominant role for the properties of the scalars, so we refer to Ref. [32] for exact formulae of the relevant cross sections and discussions of direct detection signals etc. Additional work on $B-L$ in connection with dark matter has been done in Refs. [16, 34].

Values around $M_{\chi} \sim 100 \mathrm{GeV}$ are an interesting limiting case for collider searches. However, since $\chi$, $N_{i}, Z^{\prime}$, and $\phi_{2}$ all obtain their masses from $B+3 \bar{L}$ breaking (20), we naturally would expect their masses to be similar:

$$
M_{Z^{\prime}} \sim m_{2} \sim M_{\chi} \sim M_{N_{i}} .
$$

To satisfy collider constraints and give a valid seesaw mechanism one needs the scale for these masses to be above $1-10 \mathrm{TeV}$, but it can of course be even higher. A valid relic density can be obtained yet again around the $\phi_{2}$ resonance, since we expect $\chi$ and $\phi_{2}$ to have similar masses anyway. The important annihilation channels are then $\chi \chi \rightarrow$ leptons, $W W, Z Z$ and $\phi_{1} \phi_{1}$. The latter three have a fixed ratio at the resonance, because for $m_{2} \gg m_{1}, M_{Z}$ one calculates

$$
\Gamma\left(\phi_{2} \rightarrow W^{+} W^{-}\right) \simeq 2 \Gamma\left(\phi_{2} \rightarrow Z Z\right) \simeq 2 \Gamma\left(\phi_{2} \rightarrow \phi_{1} \phi_{1}\right) \simeq \frac{m_{2}^{3}}{16 \pi v^{2}} \sin ^{2} \alpha .
$$

\footnotetext{
${ }^{3}$ Note that we need an exact $\mathbb{Z}_{2}$ for dark matter, while a valid IH solution could also work with a broken $\mathbb{Z}_{2}$. This would however necessitate a more complicated model, so Occam's razor suggests an exact $\mathbb{Z}_{2}$.
} 

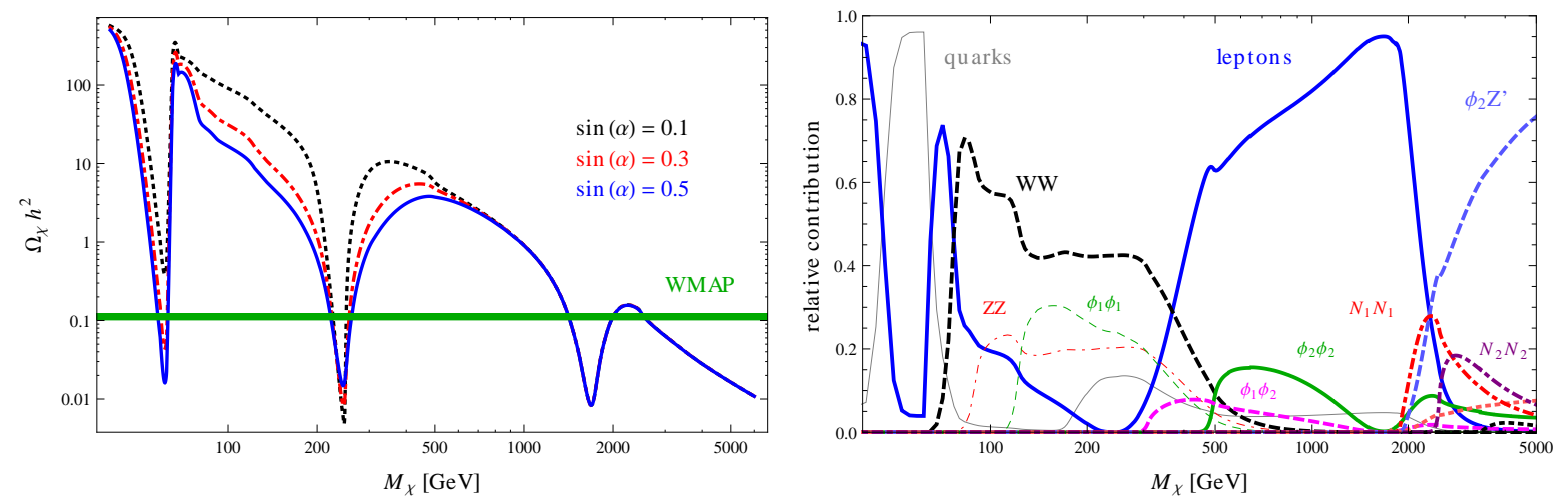

Figure 3: Left: Relic density of $\chi$ for the parameters $m_{1}=125 \mathrm{GeV}, m_{2}=500 \mathrm{GeV}, v_{S}=2.3 \mathrm{TeV}, g^{\prime}=0.25$, $N_{1}=1.9 \mathrm{TeV}, N_{2}=2.5 \mathrm{TeV}$, and $\sin \alpha=0.5$ (blue), 0.3 (red) and 0.1 (black). This puts the $\phi_{1}, \phi_{2}$ and $Z^{\prime}$ resonances at $\sim 60 \mathrm{GeV}, 250 \mathrm{GeV}$ and $1.7 \mathrm{TeV}$, respectively. The green band shows the $3 \sigma$ range measured by WMAP. Right: Relative contribution to the relic density by the processes $\chi \chi \rightarrow q \bar{q}$ (sum over all quarks), leptons (including neutrinos), $Z Z$, etc., for $\sin \alpha=0.3$.

For $M_{\chi} \simeq m_{2} / 2>m_{t}$ there is of course the additional important decay into top quarks. However, for a DM candidate this heavy, we also have a $Z^{\prime}$ resonance $M_{\chi} \simeq M_{Z^{\prime}} / 2$ independent of the mixing angle $\alpha$. Due to the different coupling of our $Z^{\prime}$ compared to $B-L$, this $Z^{\prime}$ resonance is particularly interesting to distinguish the models. The interactions between fermions and $Z^{\prime}$ are given by

$$
\begin{aligned}
\mathcal{L} \supset g^{\prime} Z_{\mu}^{\prime}( & -\frac{3}{2} \bar{\chi} \gamma^{\mu} \gamma_{5} \chi+\frac{1}{3} \sum_{q} \bar{q} \gamma^{\mu} q-3 \bar{e} \gamma^{\mu} e+3 \bar{\tau} \gamma^{\mu} \tau \\
& \left.+\frac{3}{2} \bar{\nu}_{e} \gamma^{\mu}\left(-\gamma_{5}\right) \nu_{e}-\frac{3}{2} \bar{\nu}_{\tau} \gamma^{\mu}\left(-\gamma_{5}\right) \nu_{\tau}+\frac{3}{2} \bar{N}_{1} \gamma^{\mu}\left(+\gamma_{5}\right) N_{1}+\ldots\right),
\end{aligned}
$$

where $\chi$ and the neutrinos are written as Majorana fermions. The structure of the effective operators $\bar{\chi} \gamma^{\mu} \gamma_{5} \chi \bar{f} \gamma_{\mu} f$ upon integrating out $Z^{\prime}$ leads to spin-independent (SI) and spin-dependent (SD) interactions in the non-relativistic limit, suppressed by $v^{2}$ (velocity) and $q^{2}$ (momentum transfer), respectively, as discussed in Ref. [35].

Around the $Z^{\prime}$ resonance, the relevant processes $\chi \chi \rightarrow Z^{\prime} \rightarrow f \bar{f}$ lead to the thermally averaged cross section $\langle\sigma v\rangle \simeq a+b v^{2}$ with $a=0$ and

$$
b \simeq \frac{2 g^{\prime 4}}{3 \pi} \frac{M_{\chi}^{2}}{\left(M_{Z^{\prime}}^{2}-4 M_{\chi}^{2}\right)^{2}+\Gamma_{Z^{\prime}}^{2} M_{Z^{\prime}}^{2}} \sum_{f}{Y^{\prime}}_{f}^{\prime 2} Y_{\chi}^{\prime 2},
$$

where we neglected the fermion masses for simplicity. This can be used to calculate the freeze-out temperature and the relic density $\Omega_{\chi} h^{2} \sim 1 / b[32]$ of $\chi$. Due to the larger coupling of $Z^{\prime}$ to leptons compared to $B-L$, the annihilation channels around the $Z^{\prime}$ resonance are mainly $\ell \bar{\ell}, \nu \bar{\nu}$, and also $N_{i} N_{i}$ if $M_{N_{i}} \lesssim M_{Z^{\prime}} / 2$. At this point it matters whether we take $\chi$ from Sec. IIIB or Sec. IIIC, because the models differ in the number of heavy neutrinos. However, additional right-handed neutrinos do not change the discussion qualitatively, so we will perform our calculations with $n_{N}=3$ (Sec. IIIB) for simplicity. In Fig. 3 we already showed the relic density of $\chi$ and the contributing processes around the $Z^{\prime}$ resonance.

While it is clear from Fig. 3 that the $Z^{\prime}$ channel can lead to the proper relic density (even for $\sin \alpha=0$ ), direct detection signals from $Z^{\prime}$ interactions are difficult to measure due to the Lorentz structure of the effective operator $\bar{\chi} \gamma^{\mu} \gamma_{5} \chi \bar{f} \gamma_{\mu} f$. Since direct detection occurs via $t$-channel $Z^{\prime}$ exchange, there is no resonance boost like in the annihilation case. The SD operators $\bar{\chi} \gamma^{\mu} \gamma_{5} \chi \bar{f} \gamma_{\mu} \gamma_{5} f$-which do not suffer from $q^{2}$ or $v^{2}$ suppression - can only be obtained via electroweak loops or $Z-Z^{\prime}$ mixing, which once again suppresses them. Correspondingly, direct detection experiments will not be sensitive to $Z^{\prime}$ exchange, so the cross section will be dominated by the scalar-induced operator $\bar{\chi} \chi \bar{q} q$, which gives SI cross sections proportional to $\sin ^{2} 2 \alpha M_{\chi}^{2} / v_{S}^{2}$. We show the cross sections for $\chi p \rightarrow \chi p$ in Fig. 团 (as calculated with microMEGAs) for the same parameters as in Fig. 3. The right relic density can be obtained for example 


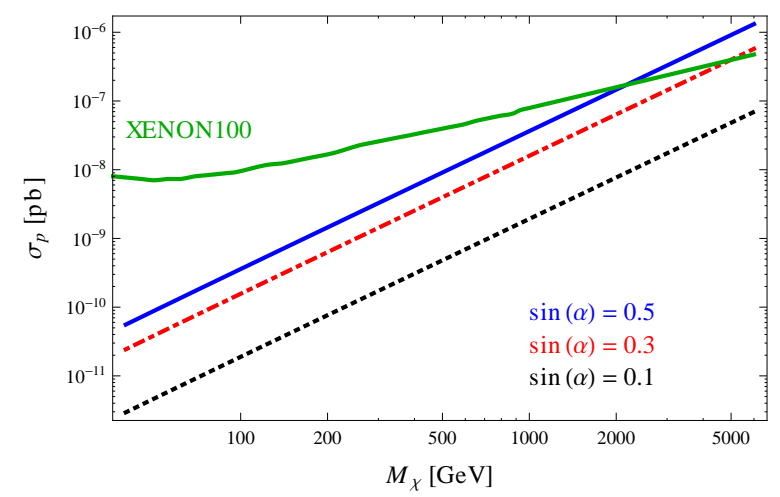

Figure 4: Spin-independent cross section of $\chi$ with a proton with the same parameters as Fig. 3, i.e. $m_{1}=125 \mathrm{GeV}$, $m_{2}=500 \mathrm{GeV}, v_{S}=2.3 \mathrm{TeV}, g^{\prime}=0.25, N_{1}=1.9 \mathrm{TeV}, N_{2}=2.5 \mathrm{TeV}$, and $\sin \alpha=0.5$ (blue), 0.3 (red, dashed) and 0.1 (black, dotted). Also shown is the XENON100 90\% C.L. exclusion from Ref. 36].

at the $\phi_{2}$ resonance with $M_{\chi} \simeq 225 \mathrm{GeV}$, which gives a cross section $\sigma_{p} / \sin ^{2} 2 \alpha \simeq 2.5 \times 10^{-9} \mathrm{pb}$. This evades current XENON100 bounds [36] but can be probed in future experiments like XENON1T [32].

We note that a supersymmetric extension of this model might result in $\alpha \ll 1$-making the $Z^{\prime}$ resonance crucial for relic abundance - similar to a supersymmetric extension of the $B-L$ model of Ref. [32] discussed in Ref. [16].

In any case, dark matter experiments will not be able to distinguish between the various $B-\sum_{\ell} x_{\ell} L_{\ell}$ models. This has to be done in collider experiments or with precision observables such as magnetic moments.

\section{CONCLUSION}

We presented the minimal model for a local $B+3\left(L_{e}-L_{\mu}-L_{\tau}\right)$ symmetry. Direct detection limits demand a breaking scale of at least $1-10 \mathrm{TeV}$ and to cancel anomalies we need to introduce right-handed neutrinos. Correspondingly, we identify the breaking scale with the seesaw scale (in fact, slightly below) and obtain low energy neutrino mass matrices that approximately conserve $L_{e}$ or $L_{e}-L_{\mu}-L_{\tau}$, which are the championed symmetries behind normal and inverted hierarchy, respectively. The latter can be obtained if a $\mathbb{Z}_{2}$ symmetry is added to the model, resulting in a stable, heavy right-handed neutrino which serves as dark matter. We stress that the $\mathbb{Z}_{2}$ is introduced to obtain the flavor structure associated with the inverted hierarchy, the DM stability is somewhat accidental. The heavy gauge boson $Z^{\prime}$ and the leftover scalar from spontaneous $B+3\left(L_{e}-L_{\mu}-L_{\tau}\right)$ breaking are the only mediators to the DM sector and are in principle observable at the LHC. Depending on the number $n_{N}$ of right-handed neutrinos, our model can produce $\theta_{13}=0\left(n_{N}=3\right.$ plus $\left.\mathbb{Z}_{2}\right)$ or $\theta_{13} \neq 0\left(n_{N} \geq 5\right.$ (odd) plus $\left.\mathbb{Z}_{2}\right)$, making $\theta_{13}$ a parameter to test the number of right-handed neutrinos in our model.

The dark matter candidate $\chi$ interacts with the Standard Model via scalar mixing and $Z^{\prime}$; the measured relic abundance can be obtained around any of the $s$-channel resonances $M_{\chi} \simeq m_{i} / 2, M_{Z^{\prime}} / 2$. The $Z^{\prime}$ contribution to direct detection measurements is highly suppressed due to the Lorentz-structure of the effective operator $\bar{\chi} \gamma^{\mu} \gamma_{5} \chi \bar{f} \gamma_{\mu} f$, so direct detection cross sections are dominated by scalar exchange and can be probed in future experiments.

Many of the phenomenological aspects of our models are very similar to previously discussed $B-L$ analyses. However, the fact that our modified gauge group includes flavor information, makes it possible to provide predictions on neutrino mixing and mass spectrum, which is impossible in theories based on $B-L$.

\section{Acknowledgments}

This work was supported by the ERC under the Starting Grant MANITOP. J.H. acknowledges support by the IMPRS for Precision Tests of Fundamental Symmetries and thanks the theory group of Kanazawa 
University, where part of this work was performed, for very kind hospitality.

\section{Appendix A: Triangle Anomalies}

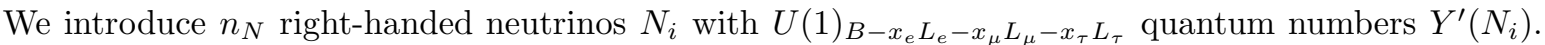
The gauge group representations of the first-generation fermions are shown in Tab. III for the second and third generation $x_{e}$ has to be replaced by $x_{\mu}$ and $x_{\tau}$ respectively.

$$
\begin{array}{|l|l|l|}
\hline L_{e}=\left(\begin{array}{l}
\nu \\
e
\end{array}\right)_{L} \sim(\mathbf{1}, \mathbf{2},-1)\left(-x_{e}\right) & e_{R}^{c} \sim(\mathbf{1}, \mathbf{1},+2)\left(x_{e}\right) & N_{i}^{c} \sim(\mathbf{1}, \mathbf{1}, 0)\left(Y^{\prime}\left(N_{i}^{c}\right)\right) \\
\hline Q_{L}^{u}=\left(\begin{array}{l}
u \\
d
\end{array}\right)_{L} \sim\left(\mathbf{3}, \mathbf{2},+\frac{1}{3}\right)\left(+\frac{1}{3}\right) & u_{R}^{c} \sim\left(\overline{\mathbf{3}}, \mathbf{1},-\frac{4}{3}\right)\left(-\frac{1}{3}\right) & d_{R}^{c} \sim\left(\overline{\mathbf{3}}, \mathbf{1},+\frac{2}{3}\right)\left(-\frac{1}{3}\right) \\
\hline
\end{array}
$$

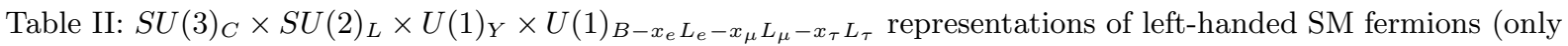
first generation shown) and right-handed neutrinos $N_{i}$.

Defining for simplicity $Y^{\prime} \equiv B-x_{e} L_{e}-x_{\mu} L_{\mu}-x_{\tau} L_{\tau}$ and $U(1)^{\prime} \equiv U(1)_{Y^{\prime}}$ we can calculate the triangle anomalies of the model [9, 37]:

$$
\begin{aligned}
& U(1)^{\prime}-\operatorname{grav}-\operatorname{grav}: \sum Y^{\prime}=N_{g} N_{c}\left(2\left(\frac{1}{3}\right)-\left(\frac{1}{3}\right)-\left(\frac{1}{3}\right)\right)+\sum_{\ell}\left(x_{\ell}-2 \cdot x_{\ell}\right)+\sum_{i} Y^{\prime}\left(N_{i}^{c}\right) \\
& =\sum_{i} Y^{\prime}\left(N_{i}^{c}\right)-\sum_{\ell} x_{\ell} \\
& U(1)^{\prime}-U(1)^{\prime}-U(1)^{\prime}: \sum Y^{\prime 3}=\sum_{i} Y^{\prime 3}\left(N_{i}^{c}\right)-\sum_{\ell} x_{\ell}^{3}, \\
& U(1)^{\prime}-U(1)^{\prime}-U(1)_{Y}: \sum Y^{\prime 2} Y=N_{g} N_{c}\left(2\left(\frac{1}{3}\right)^{2}\left(\frac{1}{3}\right)+\left(-\frac{1}{3}\right)^{2}\left(-\frac{4}{3}\right)^{2}\left(-\frac{1}{3}\right)^{2}\left(\frac{2}{3}\right)\right)=0, \\
& U(1)^{\prime}-U(1)_{Y}-U(1)_{Y}: \sum_{\ell} Y^{\prime} Y^{2}=N_{g} N_{c}\left(2\left(\frac{1}{3}\right)\left(\frac{1}{3}\right)^{2}+\left(-\frac{1}{3}\right)\left(-\frac{4}{3}\right)^{2}+\left(-\frac{1}{3}\right)\left(\frac{2}{3}\right)^{2}\right) \\
& \quad+\sum_{\ell}\left(2^{2} x_{\ell}-2 x_{\ell}\right)=-2 N_{g} N_{c}\left(\frac{1}{3}\right)+2 \sum_{\ell} x_{\ell}, \\
& U(1)^{\prime}-S U(3)-S U(3): \sum_{\mathbf{3}, \overline{3}} Y^{\prime}=N_{g} N_{c}\left(2\left(\frac{1}{3}\right)-\left(\frac{1}{3}\right)-\left(\frac{1}{3}\right)\right)=0, \\
& U(1)^{\prime}-S U(2)-S U(2): \sum_{\mathbf{2}} Y^{\prime}=2 N_{g} N_{c}\left(\frac{1}{3}\right)-2 \sum_{\ell} x_{\ell},
\end{aligned}
$$

where we introduced the number of generations $N_{g}=3$ and the number of colors $N_{c}=3$. Thus the conditions for anomaly-freedom are

$$
\sum_{i}^{n_{N}} Y^{\prime}\left(N_{i}^{c}\right)=\sum_{\ell} x_{\ell}=3, \quad \text { and } \quad \sum_{i} Y^{\prime 3}\left(N_{i}^{c}\right)=\sum_{\ell} x_{\ell}^{3} \text {. }
$$

In this paper we discuss the choice $x_{e}=-x_{\mu}=-x_{\tau}=-3$, which leads to the conditions for the right-handed neutrino charges

$$
\sum_{i}^{n_{N}} Y^{\prime}\left(N_{i}\right)=-3, \quad \text { and } \quad \sum_{i} Y^{\prime 3}\left(N_{i}\right)=-3^{3}
$$




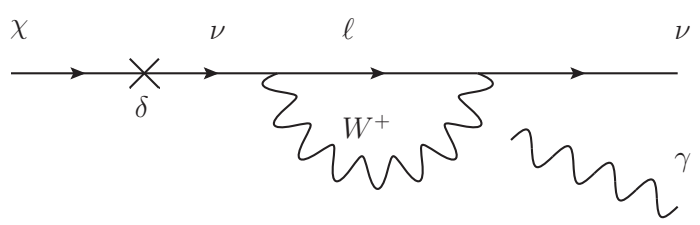

Figure 5: Decay of the dark matter candidate $\chi$ due to small $Z_{2}$ breaking $\varepsilon$ in the Dirac mass matrix $m_{D}$.

\section{Appendix B: Unstable dark matter from $\mathbb{Z}_{2}$ Breaking}

We will discuss the connection between the lifetime of $\chi \equiv N_{3}$ and $\theta_{13}$ in the model of Sec. IIIB. as they are both connected to $\mathbb{Z}_{2}$ breaking. $\mathbb{Z}_{2}$ breaking in $\mathcal{M}_{R}$ does not induce a non-zero $\theta_{13}$, so we assume there are breaking terms in $m_{D}:^{4}$

$$
\mathcal{L} \supset \delta \bar{\nu}_{\mu} \chi+\varepsilon \bar{\nu}_{\tau} \chi+\text { h.c. }
$$

where $\delta$ and $\varepsilon$ carry no $B+3 \bar{L}$ charge, wlog. The mass matrix $\mathcal{M}_{\nu}$ from Eq. (9) gets perturbed by

$$
\Delta \mathcal{M}=-\left(\begin{array}{ccc}
0 & 0 & 0 \\
\cdot & \delta^{2} / M_{\chi} & \delta \varepsilon / M_{\chi} \\
\cdot & \cdot & \varepsilon^{2} / M_{\chi}
\end{array}\right)
$$

The lowest neutrino mass is no longer zero but rather $\simeq(\delta-\varepsilon)^{2} / 2 M_{\chi}$, and furthermore

$$
\sin \theta_{13} \simeq \frac{\delta^{2}-\varepsilon^{2}}{M_{\chi}} \frac{R}{\sqrt{18}} \frac{1}{\sqrt{\left|\Delta m_{31}^{2}\right|}},
$$

with $R \equiv \Delta m_{21}^{2} / \Delta m_{31}^{2} \simeq 0.03$.

The Lagrangian (B1) generates the $\chi$ decay via $W$-loop (Fig. 5) and tree-level $\chi \rightarrow \nu Z \rightarrow 3 \nu$, the latter of which dominates and can be estimated via

$$
\Gamma(\chi \rightarrow 3 \nu) \simeq \frac{G_{F}^{2}}{192 \pi^{3}}\left(\frac{\delta}{M_{\chi}}\right)^{2} M_{\chi}^{5},
$$

where we set $\varepsilon=0$ for simplicity. The decay width is therefore linear in $\sin \theta_{13}$ :

$$
\begin{aligned}
\Gamma(\chi \rightarrow 3 \nu) & \simeq \sqrt{18} \frac{G_{F}^{2}}{192 \pi^{3}} \frac{\sqrt{\left|\Delta m_{31}^{2}\right|}}{R} M_{\chi}^{4} \sin \theta_{13} \\
& \simeq \sin \theta_{13}\left(\frac{M_{\chi}}{100 \mathrm{GeV}}\right)^{4} 10^{-14} \mathrm{GeV}
\end{aligned}
$$

resulting in a lifetime compared to the age of the universe

$$
\tau_{\chi} / \tau_{\text {Universe }} \simeq \frac{10^{-28}}{\sin \theta_{13}}\left(\frac{100 \mathrm{GeV}}{M_{\chi}}\right)^{4} .
$$

Even for the smallest currently allowed $\sin \theta_{13} \simeq 0.03$ (at $3 \sigma$ ) it is not possible to make $\chi$ a sufficiently long-lived cold dark matter candidate.

\footnotetext{
4 We do not specify the origin of these parameters; they can be obtained with an additional Higgs doublet or as effective operators from $\mathbb{Z}_{2}$ breaking at the Planck scale. In the former case the additional scalars might contribute to the width of $\chi$.
} 


\section{Appendix C: Majorana Interactions}

Here we provide some details on the rewriting of the Lagrangian of the right-handed Dirac fermion $N_{3}$ in terms of a Majorana fermion $\chi$. After spontaneous breaking of $B+3 \bar{L}$ we have

$$
\begin{aligned}
\mathcal{L}_{N_{3}} & =i \overline{N_{3}} \gamma^{\mu}\left(\partial_{\mu}-i(-3) g^{\prime} Z_{\mu}^{\prime}\right) P_{R} N_{3}-Y_{\chi} S{\overline{N_{3}}}^{c} P_{R} N_{3}-Y_{\chi} \bar{S} \overline{N_{3}} P_{L} N_{3}^{c} \\
& =i \overline{N_{3}} \gamma^{\mu}\left(\partial_{\mu}-i(-3) g^{\prime} Z_{\mu}^{\prime}\right) P_{R} N_{3}-Y_{\chi} \frac{v_{S}}{\sqrt{2}}\left(\overline{N_{3}} P_{R} N_{3}+\overline{N_{3}} P_{L} N_{3}^{c}\right)\left(1+\frac{s}{v_{S}}\right) .
\end{aligned}
$$

Introducing two Majorana fields $\phi=\phi^{c}$ and $\chi=\chi^{c}$ so that $N_{3}=P_{L} \phi+P_{R} \chi$ gives

$$
\mathcal{L}_{\chi}=\frac{i}{2} \chi^{T} \mathcal{C} \gamma^{\mu} \partial_{\mu} \chi-\frac{3}{2} g^{\prime} Z_{\mu}^{\prime} \chi^{T} \mathcal{C} \gamma^{\mu} \gamma_{5} \chi-Y_{\chi} \frac{v_{S}}{\sqrt{2}} \chi^{T} \mathcal{C} \chi\left(1+\frac{s}{v_{S}}\right)
$$

where we omitted the non-interacting $\phi$ and used the Majorana identities ${ }^{5}$

$$
\bar{\chi} \gamma^{\mu} \chi=0=\bar{\chi} \gamma_{\mu} \gamma^{5} \partial^{\mu} \chi-\left(\partial^{\mu} \bar{\chi}\right) \gamma_{\mu} \gamma^{5} \chi
$$

as well as the general equations $\left[\mathcal{C}, \gamma_{5}\right]=0, \overline{\mathcal{C}}=\mathcal{C}$ and $\bar{P}_{L, R}=P_{R, L}$. Due to the Majorana condition we also have $\chi^{T} \mathcal{C}=\bar{\chi}$. Reading off the mass $m_{\chi} / 2=Y_{\chi} v_{S} / \sqrt{2}$ we note that $\chi$ is to be treated as a real field when computing functional derivatives (additional factor of 2 ).

A similar analysis can be performed for the neutrinos (active and right-handed), with the additional complication of mixing (via seesaw). The only important part is actually the $\gamma_{5}$ in the $Z^{\prime}$ and $Z$ interactions, which leads to spin-dependent scattering. For the active neutrinos the $\gamma_{5}$ stems from a left-handed projector, so we end up with

$$
\begin{aligned}
\mathcal{L} \supset & +\frac{1}{2}\left(+3 g^{\prime}\right) Z_{\mu}^{\prime} \bar{\nu}_{e} \gamma^{\mu}\left(-\gamma_{5}\right) \nu_{e}+\frac{1}{2}\left(-3 g^{\prime}\right) Z_{\mu}^{\prime} \bar{\nu}_{\tau} \gamma^{\mu}\left(-\gamma_{5}\right) \nu_{\tau} \\
& +\frac{1}{2}\left(+3 g^{\prime}\right) Z_{\mu}^{\prime} \bar{N}_{1} \gamma^{\mu}\left(+\gamma_{5}\right) N_{1}+\frac{1}{2}\left(-3 g^{\prime}\right) Z_{\mu}^{\prime} \bar{N}_{2} \gamma^{\mu}\left(+\gamma_{5}\right) N_{2},
\end{aligned}
$$

etc., where all fermions are Majorana particles.

[1] S. Choubey and W. Rodejohann, Eur. Phys. J. C 40, 259 (2005) arXiv:hep-ph/0411190.

[2] R. Foot, Mod. Phys. Lett. A 6, 527 (1991);

X. G. He, G. C. Joshi, H. Lew and R. R. Volkas, Phys. Rev. D 44, 2118 (1991);

R. Foot, X. G. He, H. Lew and R. R. Volkas, Phys. Rev. D 50, 4571 (1994) arXiv:hep-ph/9401250.

[3] For a collection of references see J. Heeck and W. Rodejohann, Phys. Rev. D 84, 075007 arXiv:1107.5238 [hep-ph]].

[4] S. T. Petcov, Phys. Lett. B 110, 245 (1982);

C. N. Leung and S. T. Petcov, Phys. Lett. B 125, 461 (1983);

G. C. Branco, W. Grimus and L. Lavoura, Nucl. Phys. B 312, 492 (1989);

A. S. Joshipura and S. D. Rindani, Eur. Phys. J. C 14, 85 (2000) arXiv:hep-ph/9811252;

R. N. Mohapatra, A. Perez-Lorenzana and C. A. de Sousa Pires, Phys. Lett. B 474, 355 (2000) arXiv:hep-ph/9911395;

L. Lavoura, Phys. Rev. D 62, 093011 (2000) arXiv:hep-ph/0005321;

K. S. Babu and R. N. Mohapatra, Phys. Lett. B 532, 77 (2002) arXiv:hep-ph/0201176;

H. J. He, D. A. Dicus and J. N. Ng, Phys. Lett. B 536, 83 (2002) arXiv:hep-ph/0203237;

S. T. Petcov and W. Rodejohann, Phys. Rev. D 71, 073002 (2005) arXiv:hep-ph/0409135;

G. Altarelli and R. Franceschini, JHEP 0603, 047 (2006) arXiv:hep-ph/0512202;

D. Meloni, JHEP 1202, 090 (2012) arXiv:1110.5210 [hep-ph]].

[5] R. Barbieri, L. J. Hall, D. Tucker-Smith, A. Strumia and N. Weiner, JHEP 9812, 017 (1998) arXiv:hep-ph/9807235;

W. Grimus and L. Lavoura, JHEP 0107, 045 (2001) arXiv:hep-ph/0105212.

\footnotetext{
${ }^{5}$ It proves convenient to add a total derivative to the Lagrangian to replace the kinetic term $\overline{N_{3}} \not \partial N_{3}$ by $\frac{1}{2}\left(\overline{N_{3}} \not \partial N_{3}-\right.$ $\left.\left(\partial^{\mu} \overline{N_{3}}\right) \gamma_{\mu} N_{3}\right)$.
} 
[6] L. Lavoura and W. Grimus, JHEP 0009, 007 (2000) arXiv:hep-ph/0008020;

W. Grimus and L. Lavoura, J. Phys. G 31, 683 (2005) arXiv:hep-ph/0410279].

[7] Q. Shafi and Z. Tavartkiladze, Phys. Lett. B 482, 145 (2000) arXiv:hep-ph/0002150;

K. S. Babu, A. G. Bachri and Z. Tavartkiladze, Int. J. Mod. Phys. A 23, 1679 (2008) arXiv:0705.4419 [hep-ph]].

[8] E. Ma, D. P. Roy and U. Sarkar, Phys. Lett. B 444, 391 (1998) arXiv:hep-ph/9810309.

[9] E. Ma, Phys. Lett. B 433, 74 (1998) arXiv:hep-ph/9709474;

E. Ma and D. P. Roy, Phys. Rev. D 58, 095005 (1998) arXiv:hep-ph/9806210;

E. Ma and U. Sarkar, Phys. Lett. B 439, 95 (1998) arXiv:hep-ph/9807307;

E. Ma and D. P. Roy, Phys. Rev. D 59, 097702 (1999) arXiv:hep-ph/9811266.

[10] L. N. Chang, O. Lebedev, W. Loinaz and T. Takeuchi, Phys. Rev. D 63, 074013 (2001) arXiv:hep-ph/0010118.

[11] H. Davoudiasl, H. S. Lee and W. J. Marciano, Phys. Rev. D 84, 013009 (2011) arXiv:1102.5352 [hep-ph]].

[12] E. Salvioni, A. Strumia, G. Villadoro and F. Zwirner, JHEP 1003, 010 (2010) arXiv:0911.1450 [hep-ph]].

[13] M. C. Chen and J. Huang, Mod. Phys. Lett. A 26, 1147 (2011) arXiv:1105.3188 [hep-ph]].

[14] H. S. Lee and E. Ma, Phys. Lett. B 688, 319 (2010) arXiv:1001.0768 [hep-ph]].

[15] S. Khalil and A. Masiero, Phys. Lett. B 665, 374 (2008) arXiv:0710.3525 [hep-ph]]; P. Fileviez Perez and S. Spinner, Phys. Rev. D 83, 035004 (2011) arXiv:1005.4930 [hep-ph]];

B. O'Leary, W. Porod and F. Staub, JHEP 1205, 042 (2012) arXiv:1112.4600 [hep-ph]].

[16] Z. M. Burell and N. Okada, Phys. Rev. D 85, 055011 (2012) arXiv:1111.1789 [hep-ph]].

[17] J. Heeck and W. Rodejohann, JHEP 1202, 094 (2012) arXiv:1112.3628 [hep-ph]].

[18] T. Schwetz, M. Tortola and J. W. F. Valle, New J. Phys. 13, 063004 (2011) arXiv:1103.0734 [hep-ph]]; T. Schwetz, M. Tortola and J. W. F. Valle, New J. Phys. 13, 109401 (2011) arXiv:1108.1376 [hep-ph]]; G. L. Fogli, E. Lisi, A. Marrone, A. Palazzo and A. M. Rotunno, Phys. Rev. D 84, 053007 (2011) arXiv:1106.6028 [hep-ph]].

[19] K. Abe et al. [T2K Collaboration], Phys. Rev. Lett. 107, 041801 (2011) arXiv:1106.2822 [hep-ex]].

[20] Y. Abe et al. [Double Chooz Collaboration], Phys. Rev. Lett. 108, 131801 (2012) arXiv:1112.6353 [hep-ex]].

[21] F. P. An et al. [Daya Bay Collaboration], Phys. Rev. Lett. 108, 171803 (2012) arXiv:1203.1669 [hep-ex]].

[22] J. K. Ahn et al. [RENO Collaboration], Phys. Rev. Lett. 108, 191802 (2012) arXiv:1204.0626 [hep-ex]].

[23] S. Goswami and A. Watanabe, Phys. Rev. D 79, 033004 (2009) arXiv:0807.3438 [hep-ph]].

[24] R. N. Mohapatra and W. Rodejohann, Phys. Lett. B 644, 59 (2007) arXiv:hep-ph/0608111;

A. Blum, R. N. Mohapatra and W. Rodejohann, Phys. Rev. D 76, 053003 (2007) arXiv:0706.3801 [hep-ph]].

[25] S. Ray, W. Rodejohann and M. A. Schmidt, Phys. Rev. D 83, 033002 (2011) arXiv:1010.1206 [hep-ph]].

[26] B. Holdom, Phys. Lett. B 166, 196 (1986); Phys. Lett. B 259, 329 (1991).

[27] P. Langacker, Rev. Mod. Phys. 81, 1199 (2009) arXiv:0801.1345 [hep-ph]].

[28] J. Erler, P. Langacker, Phys. Lett. B 456, 68-76 (1999) arXiv:hep-ph/9903476]; J. Erler, arXiv:hep-ph/0005084.

[29] [The LEP Collaborations: ALEPH Collaboration, DELPHI Collaboration, L3 Collaboration, OPAL Collaboration, the LEP Electroweak Working Group, the SLD Electroweak, Heavy Flavour Groups], arXiv:hep-ex/0312023

M. S. Carena, A. Daleo, B. A. Dobrescu and T. M. P. Tait, Phys. Rev. D 70, 093009 (2004) arXiv:hep-ph/0408098.

[30] L. Basso, A. Belyaev, S. Moretti and C. H. Shepherd-Themistocleous, Phys. Rev. D 80, 055030 (2009) arXiv:0812.4313 [hep-ph]];

L. Basso, S. Moretti and G. M. Pruna, Phys. Rev. D 83, 055014 (2011) arXiv:1011.2612 [hep-ph]].

[31] E. Komatsu et al. [WMAP Collaboration], Astrophys. J. Suppl. 192, 18 (2011) arXiv:1001.4538 [astroph.CO]];

D. Larson et al., Astrophys. J. Suppl. 192, 16 (2011) arXiv:1001.4635 [astro-ph.CO]].

[32] N. Okada and O. Seto, Phys. Rev. D 82, 023507 (2010) arXiv:1002.2525 [hep-ph]];

S. Kanemura, O. Seto and T. Shimomura, Phys. Rev. D 84, 016004 (2011) arXiv:1101.5713 [hep-ph]].

[33] G. Belanger, F. Boudjema, A. Pukhov and A. Semenov, Comput. Phys. Commun. 176, 367 (2007) arXiv:hep-ph/0607059;

G. Belanger, F. Boudjema, A. Pukhov and A. Semenov, Comput. Phys. Commun. 180, 747 (2009) arXiv:0803.2360 [hep-ph]];

G. Belanger, F. Boudjema, A. Pukhov and A. Semenov, arXiv:1005.4133 [hep-ph].

[34] S. Kanemura, T. Nabeshima and H. Sugiyama, Phys. Rev. D 85, 033004 (2012) arXiv:1111.0599 [hep-ph]]; N. Okada and Y. Orikasa, arXiv:1202.1405 [hep-ph].

[35] M. Freytsis and Z. Ligeti, Phys. Rev. D 83, 115009 (2011) arXiv:1012.5317 [hep-ph]].

[36] E. Aprile et al. [XENON100 Collaboration], Phys. Rev. Lett. 107, 131302 (2011) arXiv:1104.2549 [astroph.CO]].

[37] C. Q. Geng and R. E. Marshak, Phys. Rev. D 39, 693 (1989). 